\title{
COMPORTAMIENTO DE VARIEDADES DE CHILE DULCE (Capsicum annum) EN LA REGIÓN OCCIDENTAL DE EL SALVADOR1
}

\author{
Lesser Linares $^{2}$
}

\begin{abstract}
RESUMEN
Comportamiento de variedades de chile dulce (Capsicun annum) en la región occidental de EI Salvador. Con el objetivo de seleccionar material vegetativo adaptable a las diferentes condiciones agroecológicas de El Salvador, se implementó una investigación de seis variedades de chile dulce. Los ensayos se realizaron en Candelaria de la Frontera, Atiquizaya y Chalchuapa en el año 2002. El diseño experimental fue de bloques al azar, con cuatro repeticiones y seis tratamientos, conformados por los variedades Nathalie, Comandante, Lido, Tikal, Quetzal y una variedad Criolla. Hubo diferencias significativas al $1 \%$ de probabilidad para las variables de altura de planta, largo, peso, diámetro, numero de frutos y rendimiento, en el combinado de las tres localidades. El material Nathalie, sobresalió en las variables altura de planta $(0,49 \mathrm{~m})$, número de frutos (259.500 frutos/ha), y rendimiento $(25,11 \mathrm{t} / \mathrm{ha})$, y como consecuencia reportó el mejor ingreso $(\$ 19.332,80)$ y la mejor relación beneficio costo $(3,31)$. La duración de anaquel varió entre seis y nueve días con respecto a los diferentes materiales de chile dulce. Los resultados obtenidos podrían crear la base para validar el cultivar Nathalie en las zonas de influencia.
\end{abstract}

\begin{abstract}
Behavior of pepper (Capsicum annun) varieties in the western region of El Salvador. An experiment was conducted, with six pepper (Capsicum annun) varieties, in order to select materials suitable for the different agroecological conditions of El Salvador. The trials were conducted in Candelaria de la Frontera, Atiquizaya y Chalchuapa. A Completely Randomized Experimental Block Design with four replications and six treatments was used, composed by the varieties Nathalie, Comandante, Lido, Tikal, Quetzal and a local variety as control. The variance analysis showed significant differences for the following variables: plant height, length, weight, diameter, number of fruits and yield, in the combination of the three localities. The Nathalie variety stood out for the variables of plant height (0.49 m), number of fruits (259,500 fruits/ha), yield (25.11 $\mathrm{t} / \mathrm{ha}$ ) and therefore, it had the highest income (\$19,332.80) and the best cost-benefit ratio (3.31). The shelf-life varied from six to nine days with respect to the different pepper materials. The results could create the base for promoting the Nathalie cultivar in the areas of influence.
\end{abstract}

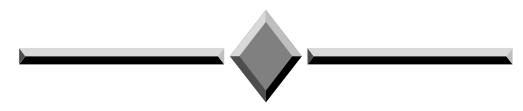

\section{INTRODUCCIÓN}

La producción de chile dulce en El Salvador, se ve afectada debido al ataque de diferentes plagas y enfermedades, a las que la mayoría de variedades actuales son susceptibles. Esto se debe al uso continuo de variedades tradicionales por parte del productor. Dentro de las alternativas de manejo, se tiene el manejo integrado de plagas con la implementación del uso de variedades de alto valor genético, tolerantes a diferentes enfermedades y mejores rendimientos. El Salvador importa al año, un promedio de 1.800 toneladas de chile dulce. En los últimos cinco años, las importaciones fueron de $\$$ 514,286 (DGEA 2002). Esta investigación permitió seleccionar materiales que se adapten a las zonas agroecológicas de producción de chile dulce del país.

\footnotetext{
1 Recibido para publicación el 18 de agosto del 2003.

2 Investigador del programa de hortalizas de CENTA-MAG. San Salvador, El Salvador. Correo electrónico: lesserlinares@hotmail.com
} 
Existe muy poca información por zona ecológica de los rendimientos de los diferentes materiales de chile dulce que se siembran a nivel comercial. Las tecnologías usadas en este país, generalmente se enfocan en reducir costos de producción y no en los beneficios netos. Semillas S.A. (2000), indicó que distribuye materiales con alto valor genético y tolerantes a virosis, incluyendo a Tikal, Quetzal y Lido. El CATIE (1993), reportó que dentro de las tácticas de manejo integrado de plagas se tiene el manejo fitogenético, que consiste en el uso de cultivares o materiales resistentes o tolerantes a las plagas y enfermedades, que han tenido y tendrán relevancia. La DGEA (2001) ha reportado que nuestro país importa la cantidad de $3.766 \mathrm{t}$ de chile dulce $(\$ 1.121 .110$ de dólares), lo que indica que esta hortaliza ocupa el séptimo lugar de importancia. El objetivo general fue seleccionar material vegetativo de chile dulce adaptable a las diferentes zonas agroecológicas.

\section{MATERIALES Y MÉTODOS}

Los ensayos se ubicaron en tres Comunidades: en el cantón El Jute de Candelaria de la Frontera, Santa Ana; en el Cantón San Juan, Atiquizaya, Ahuachapán y en el cantón San José, Chalchuapa. La selección de los ambientes se realizó por las características climáticas y edáficas (Cuadro 1).

En la primera comunidad, la investigación fue ejecutada durante los meses de mayo a octubre de 2002.
En la segunda y tercera localidad, se inició en agosto de 2002 a enero de 2003.

El diseño empleado fue de bloques completos al azar. El ensayo constó de seis tratamientos, con cuatro repeticiones, la unidad experimental consistió de cinco surcos de plantas de $4 \mathrm{~m}$ de largo y $4 \mathrm{~m}$ de ancho, dando un área de $16,0 \mathrm{~m}^{2}$, con un distanciamiento de $1,00 \mathrm{~m}$ entre surcos y 0,50 m entre plantas, dando un total de 45 plantas. La parcela útil constó de tres surcos, con un largo de 3,0 m y un ancho de 1,5 m, cuya área fue de 4,50 $\mathrm{m}^{2}$. Para la toma de datos se emplearon cinco plantas.

El manejo agronómico se inició, con el establecimiento del semillero en bandejas y protegido con Agronet (invernadero artesanal), el manejo de malezas, fue manual durante todo el ciclo fenológico del cultivo, en el manejo de plagas, se basó en el monitoreo y muestreos de plagas y en forma especifica, para el manejo de insectos se realizaron aplicaciones de aceite de NEEM (80cc/bombada), con una frecuencia de 15 días, además se aplicó Clorfenapir, para ácaros, en el manejo de enfermedades se aplicó Clorotalonilo (80 cc/bombada) y Hidróxido de Cobre (100 g/bombada), cada 15 días, en forma alternada y según se presentaba la incidencia de las lluvias, la fertilización se basó en los resultados de análisis del suelo, se aplicó fórmula 16-20-00, al trasplante (3,5 kg/ha), y a los 15 días después de la siembra se aplicó urea $(2,8 \mathrm{~kg} / \mathrm{ha})$. Posteriormente, al momento de la primera cosecha, se aplicó la fórmula 16-20-00, a una dosis de 2,0 kg/ha, la cual se repitió cada 15 días (cada dos cosechas); en total se efectuaron 16 cosechas.

Cuadro 1. Condiciones edáficas y climáticas durante la evaluación de variedades de chile dulce en diferentes ambientes de El Salvador. El Salvador, 2003.

\begin{tabular}{|c|c|c|c|c|c|c|}
\hline Número de localidad & \multicolumn{2}{|r|}{ Uno } & \multicolumn{2}{|r|}{ Dos } & \multicolumn{2}{|r|}{ Tres } \\
\hline Departamento & & Santa Ana & & Ahuachapán & & Santa Ana \\
\hline Municipio & Cande & laria de la F. & & Atiquizaya & & Chalchuapa \\
\hline Cantón & & El Jute & & San Juan B. & & San José C. \\
\hline \multicolumn{7}{|l|}{ Climáticas * } \\
\hline Altitud (msnm) & & 680 & & 600 & & 700 \\
\hline Temperatura $\left({ }^{\circ} \mathrm{C}\right)$ & & 32 & & 30 & & 26 \\
\hline Humedad relativa $(\%)$ & & 80 & & 83 & & 81 \\
\hline Precipitación (mm) & & 1.565 & & 1.863 & & 1.820 \\
\hline \multicolumn{7}{|l|}{ Edaficas $* *$} \\
\hline Textura & Franco Arc & illo Arenosa & Fral & nco Arenosa & Fra & Inco Limoso \\
\hline Fósforo (ppm) & 16 & Alto & 24 & Alto & 15 & Alto \\
\hline Potasio (ppm) & 319 & Muy Alto & 401 & Muy Alto & 305 & Muy Alto \\
\hline $\mathrm{Ph}$ & 4,4 & Ext. Ácido & 5,1 & Mod. Ácido & 6,2 & Lig. Ácido \\
\hline Topografía & & Semiplano & & Plano & & Plano \\
\hline
\end{tabular}

* Datos proporcionados por los Técnicos de Transferencia de CENTA.

** Datos del análisis de suelo del Laboratorio de CENTA. 
Los tratamientos empleados en este ensayo fueron: $\mathrm{T} 1$ = Nathalie, $\mathrm{T} 2$ = Comandante, $\mathrm{T} 3=$ Lido, $\mathrm{T} 4=\mathrm{T}-$ kal, T5 = Quetzal, T6 = Criollo (Tres Cantos). Esto se describe en el Cuadro 2.

Las variables fueron: a) altura de planta al inicio de la cosecha, b) diámetro del fruto, c) largo del fruto, d) peso del fruto, e) número de frutos por tratamiento, f) rendimiento por hectárea, g) frutos dañados por quemadura de sol, h) beneficio por tratamiento y i) duración de anaquel por material vegetativo.

Para las variables de desarrollo de la planta y cosecha del fruto se realizó, análisis de varianza (altura planta, diámetro, largo, peso, número y rendimiento de frutos, no así para los frutos dañados por sol (solo porcentaje) y duración de anaquel (numero días), el análisis económico fue apoyado con la relación beneficios costos.

Para la parte económica se empleó la relación beneficio-costo, basado en el costo de la semilla, el costo de producción de cada repetición y posteriormente de cada tratamiento. De igual manera se obtuvo el precio de venta de los frutos comerciales, y por último el ingreso por tratamiento. El producto se vendió en el mercado local de cada comunidad, sin diferencia de precio de venta por sus características varietales.

Para el análisis estadístico se realizó el análisis de varianza; al obtenerse diferencias entre las medias, se implementó la prueba de separación de medias de Duncan.

\section{RESULTADOS Y DISCUSIÓN}

En el Cuadro 3, se presentan los datos estadísticos para las variables en estudio, así como sus medias de al- tura de planta, largo, diámetro y peso de frutos, también numero de frutos por planta y rendimiento, estos resultados del análisis estadístico son del combinado de las tres comunidades.

Para la variable altura de planta, se observó diferencia significativa al $1 \%$ entre los tratamientos. La mayor altura se obtuvo en el material de Nathalie $(49,20$ cm) y la menor altura se observó en el Criollo (34,77 $\mathrm{cm}$ ), donde Nathalie superó en un $42 \%$, al Criollo.

En relación a largo de fruto, el punto más largo fue el de Tikal $(17,88 \mathrm{~cm})$ (alargado) y el Criollo el menor $(10,12 \mathrm{~cm})$ (cónico). Esto concuerda con lo reportado por CENTA 2002, donde el largo de fruto de Tikal es de $14-20 \mathrm{~cm}$.

Para la variable diámetro de fruto el material Lido fue superior al Criollo en un $38 \%$ y al Nathalie un 22 $\%$. CENTA (2002), reportó que el diámetro de lo frutos de estos materiales, varía de $4-9 \mathrm{~cm}$, dentro de los cuales se hallaron los rangos de diámetro de los frutos cosechados.

El mejor resultado en peso del fruto, se obtuvo en Comandante que superó en un $59 \%$ al fruto del Crio1lo. Según datos de la guía de chile dulce del CENTA (2002), el peso del fruto de cosecha es de 20-95 g; para el ensayo varió menos, el promedio fue de 102,41 g.

La variable número de frutos por planta mostró el mayor valor para Nathalie (13,70 unidades) y el menor fue para el Criollo (10,30 frutos). La variable fruto por hectárea, refleja una producción de 259.500 unidades/ha para la variedad Nathalie y el material criollo con 184.000 unidades/ha, el Nathalie supera al Criollo un $41 \%$ y al Quetzal en un $11 \%$, con una diferencia significativa del $1 \%$.

Cuadro 2. Características de materiales de chile dulce cultivadas en El Salvador.

\begin{tabular}{|c|c|c|c|c|c|c|}
\hline Característica & $\begin{array}{l}\text { Híbrido } \\
\text { Nathalie }\end{array}$ & $\begin{array}{l}\text { Híbrido } \\
\text { Quetzal }\end{array}$ & $\begin{array}{c}\text { Híbrido } \\
\text { Tikal }\end{array}$ & $\begin{array}{l}\text { Híbrido } \\
\text { Lido }\end{array}$ & $\begin{array}{c}\text { Híbrido } \\
\text { Comandante }\end{array}$ & $\begin{array}{c}\text { Variedad Criollo } \\
\text { Tres Cantos }\end{array}$ \\
\hline $\begin{array}{l}\text { Tipo de } \\
\text { crecimiento }\end{array}$ & Indeterminado & Determinado & Indetermi nado & Determinado & Determinado & Determinado \\
\hline Cosecha (días) & $85-95$ & $68-70$ & $68-70$ & $80-90$ & $90-95$ & $80-90$ \\
\hline \multirow[t]{2}{*}{ Forma } & Cónica & Cónica & Alargado & Semialargado & Cónica & Cónica \\
\hline & 2-3 Lóbulos & 2-3 Lóbulos & 2-3 Lóbulos & 3-4 Lóbulos & 3-4 Lóbulos & 2-3 Lóbulos \\
\hline Tolerancia & VYP, VMT & VYP, VMT VET & VYP, VMT & ToMV, VMT & VYP, VMT & Suceptible VMT \\
\hline Rendimiento(t/ha) & $22-28$ & $22-24$ & $19-22$ & $18-20$ & $18-20$ & $14-16$ \\
\hline Fructificación & Continua & Continua & Continua & Continua & Continua & Continua \\
\hline
\end{tabular}

VYP Virus "Y" de la Papa V VMT Virus del Mosaico del tabaco VET Virus Esch del tabaco 
Cuadro 3. Características agronómicas de la planta y frutos en el ensayo de los materiales de chile dulce, Combinado de localidades, El Salvador, 2003.

\begin{tabular}{lllclcc}
\hline Material & $\begin{array}{c}\text { Altura de } \\
\text { planta (m) }\end{array}$ & $\begin{array}{c}\text { Largo de } \\
\text { fruto }(\mathbf{c m})\end{array}$ & $\begin{array}{c}\text { Diámetro de } \\
\text { fruto }(\mathbf{c m})\end{array}$ & $\begin{array}{c}\text { Peso de } \\
\text { fruto }(\mathbf{g})\end{array}$ & $\begin{array}{c}\text { No de frutos/ } \\
\text { planta }\end{array}$ & $\begin{array}{c}\text { Rendimiento } \\
(\mathbf{t} / \mathbf{h a})\end{array}$ \\
\hline Nathalie & $0,49 \mathrm{a}$ & $13,10 \mathrm{c}$ & $5,21 \mathrm{~b}$ & $96,0 \mathrm{~b}$ & $13,70 \mathrm{a}$ & $25,11 \mathrm{a}$ \\
Comandante & $0,42 \mathrm{c}$ & $10,12 \mathrm{~d}$ & $6,21 \mathrm{a}$ & $123,3 \mathrm{a}$ & $9,85 \mathrm{~d}$ & $23,44 \mathrm{a}$ \\
Lido & $0,39 \mathrm{~d}$ & $10,94 \mathrm{de}$ & $6,36 \mathrm{a}$ & $120,9 \mathrm{a}$ & $9,95 \mathrm{~d}$ & $23,41 \mathrm{a}$ \\
Tikal & $0,47 \mathrm{ab}$ & $17,88 \mathrm{a}$ & $4,68 \mathrm{c}$ & $95,9 \mathrm{c}$ & $10,55 \mathrm{c}$ & $19,77 \mathrm{~b}$ \\
Quetzal & $0,45 \mathrm{bc}$ & $14,56 \mathrm{~b}$ & $5,42 \mathrm{~b}$ & $100,9 \mathrm{~b}$ & $12,50 \mathrm{~b}$ & $23,79 \mathrm{a}$ \\
Criollo & $0,35 \mathrm{e}$ & $10,12 \mathrm{e}$ & $4,62 \mathrm{c}$ & $77,5 \mathrm{c}$ & $10,30 \mathrm{c}$ & $12,37 \mathrm{c}$ \\
$\mathrm{C}$ & 0,43 & 12,98 & 5,42 & 102,41 & 10,75 & 21,32 \\
$\mathrm{~F}$ & $* *$ & $* *$ & $* *$ & $* *$ & $* *$ & $* *$ \\
$\mathrm{Cv}(\%)$ & 6,99 & 7,23 & 5,02 & 9,82 & 4,61 & 14,06 \\
\hline
\end{tabular}

El análisis de la variable de rendimiento mostró diferencia significativa al $1 \%$. El rendimiento de Nathalie superó solo en un $6 \%$ al rendimiento de Quetzal y en un $103 \%$ al Criollo.

CENTA (2003) reportó rendimientos de 14-28 t/ha, y los promedios de cosecha se mantuvieron dentro de estos rangos, menos el Criollo con 12,37 t/ha.

\section{Datos de frutos quemados por el sol}

El número de frutos afectados por las quemaduras de sol, tuvo un rango de variación de 2,5 a 4,3\%; el valor más alto lo mostró la variedad Criolla y con un promedio de 3,09\%. El menor número de frutos quemados se observó en Quetzal, debido a la mayor cobertura del follaje.

\section{Prueba de Anaquel}

La prueba de anaquel indicó que los materiales híbridos (Nathalie, Quetzal, Comandante, Lido y Tikal) duran en promedio de 7 - 9 días y el Criollo de 6-7 días, iniciando cambio de color de la cáscara a rojo y perdien- do su turgencia. Este análisis fue con base a lecturas de tiempo (numero días) y no con análisis estadístico.

La comunidad donde se manifestaron mejor los materiales híbridos fue en Atiquizaya, Ahuchapan, y el mejor fue Nathalie en cuanto a número de frutos (274.000 por hectárea) y rendimiento $(28,67 \mathrm{t} / \mathrm{ha})$. También el peso de fruto de Comandante $(143,88 \mathrm{~g})$ y Lido (137,75 gramos). En la comunidad de El Jute, Candelaria de la Frontera, Santa Ana, fue donde menos se manifestó el potencial de genotipo de los materiales.

El análisis económico se explica en el Cuadro 4.

\section{RECOMENDACIONES}

Trabajar en el área de validación con el material de Nathalie, para divulgar sus ventajas, en relación al del número de frutos y rendimientos.

A nivel económico, llevar un ensayo donde se permita comparar los materiales Nathalie y Quetzal, con distintos niveles de fertilización del cultivo.

Cuadro 4. Análisis económico del ensayo de materiales de chile dulce, las tres localidades. El Salvador, 2003.

\begin{tabular}{lrrrrrrr}
\hline \multicolumn{1}{c}{ Factor } & Nathalie & Comandante & \multicolumn{1}{c}{ Lido } & Tikal & Quetzal & Criollo & Media \\
\hline Ingreso \$ & $19.332,8$ & $14.117,8$ & $14.341,3$ & $15.309,8$ & $17.395,8$ & $13.708,0$ & $13.645,9$ \\
Costo \$ & $4.481,0$ & $4.563,0$ & $4.563,0$ & $4.458,0$ & $4.596,0$ & $3.432,0$ & $4.348,8$ \\
Beneficio \$ & $14.851,8$ & $9.554,8$ & $9.778,3$ & $10.851,8$ & $12.799,8$ & $10.276,0$ & $9.297,1$ \\
R B/C 3,31 & 2,09 & 2,14 & 2,43 & 2,78 & 2,99 & 2,14 & \\
\hline
\end{tabular}

NOTA: El precio de venta por fruto es de $\$ 0,0745$, es un promedio ponderado, obtenido de las catorce cosechas del cultivo y vendido en el mercado local de la comunidad. 
Enfocar una investigación de mercado, para afinar la preferencia de los consumidores, y sobre todo hacia qué nicho de mercado enfocar el tipo de fruto producido por cada material evaluado.

\section{LITERATURA CITADA}

CENTRO DE AGRICULTURA TROPICAL INVESTIGACIÓN Y ENSEÑANZA (CATIE). 1993. Manejo Integrado de Chile Dulce. Costa Rica. $52 \mathrm{p}$.

MINISTERIO DE AGRICULTURA Y GANADERÍA, DIRECCIÓN GENERAL DE ECONOMÍA AGROPE-
CUARIA (DGEA). 2002. Anuario estadístico agropecuario, San salvador, El Salvador, 85 p.

CENTRO NACIONAL DE TECNOLOGÍA AGROPECUARIA Y FORESTAL (CENTA). 2002. Cultivo de chile dulce. Ministerio de Agricultura y Ganadería. San Salvador, El Salvador. 50 p.

DIRECCIÓN GENERAL DE ECONOMÍA AGROPECUARIA (DGEA). 2001. Anuario estadístico agropecuario. Ministerio de Agricultura y Ganadería. San Salvador, El Salvador, 85 p. 